\title{
Immunization of rainbow trout Oncorhynchus mykiss against Discocotyle sagittata (Monogenea)
}

\author{
Miguel Rubio-Godoy ${ }^{1, *}$, Jens Sigh $^{2}$, Kurt Buchmann², Richard C. Tinsley ${ }^{1}$ \\ ${ }^{1}$ School of Biological Sciences, University of Bristol, Woodland Road, Bristol BS8 1UG, United Kingdom \\ ${ }^{2}$ Department of Veterinary Microbiology, Section of Fish Diseases, Royal Veterinary and Agricultural University, \\ 4 Stigbøjlen, 1870 Frederiksberg C., Denmark
}

\begin{abstract}
Rainbow trout Oncorhynchus mykiss were injected intraperitoneally with 2 different Discocotyle sagittata extracts dissolved in PBS and subsequently exposed to controlled infection. Immunization resulted in significantly reduced $(p<0.0001)$ worm intensities in $>50 \%$ of vaccinated fish (response arbitrarily defined as parasite burdens < mean control intensity $-1 \mathrm{SD}$ ), irrespective of the immunization regime (different parasite extracts, dosing and application schedules) employed. The protective effect of worm extract applied in Freund's complete adjuvant (FCA) did not differ significantly from extract given in PBS. Vaccination with embryonated parasite eggs extract and with FCA alone did not result in partial immunity, suggesting the observed protective effect is specific. Immunized fish had significantly higher specific antibody titres at the time of dissection (as determined by ELISA) than both naïve and control fish. Overall, a significant negative correlation was found between antibody titres and worm burdens, suggesting immunoglobulins are implicated in mediating partial immunity. Western blot tests indicated the 2 different worm extracts used to immunize fish share antigens, but each one primarily induced recognition of a distinct band (30 and $38 \mathrm{kDa}$ ). Immunization seems to promote a shift between 2 equilibria, rather than progressively increasing protection. This would explain why boosting did not increase immunity, and why 2 different extracts primarily inducing recognition of 2 distinct antigens provide similar degrees of protection. Although several other non-specific and cellular factors are likely to be involved in controlling parasite numbers, it cannot be excluded that antibodies could be involved in mediating the observed partial immunity.
\end{abstract}

KEY WORDS: Oncorhynchus mykiss · Trout · Monogenea · Discocotyle sagittata · Immunization · Antibodies

Resale or republication not permitted without written consent of the publisher

\section{INTRODUCTION}

Fish in the wild are rarely infected with large numbers of parasites; this could be a consequence of a well developed host immune system (Woo 1992, Secombes \& Chappell 1996), in addition to the stochastic factors commonly invoked to explain naturally overdispersed parasite distributions. However, under the stressful conditions experienced by fish in intensive farms, parasitic infections leading to significant pathology and mortality do occur (Thoney \& Hargis 1991, Woo 1992). Relatively little is known about piscine innate immunity to helminths, and the immune mechanisms underlying the documented cases of host responses to parasitic infections have been insufficiently described (Buchmann 1999, Buchmann \& Lindenstrøm 2002). However, as reviewed by Buchmann et al. (2001), several investigations have demonstrated that teleosts can develop partial protective immunity against ectoparasitic monogeneans after natural infection (Jahn \& Kuhn 1932, Nigrelli \& Breder 1934, Nigrelli 1937), experimental challenges (Scott \& Robinson 1984, Scott 1985, Slotved \& Buchmann 1993, Bondad-Reantaso et al. 1995) and active immunization (Vladimirov 1971, Kim et al. 2000). 
Discocotyle sagittata is a monogenean parasite of freshwater salmonid fishes which can be an important pathogen of farmed trout (Gannicott 1997). Previous studies (Gannicott 1997, Gannicott \& Tinsley 1997, $1998 \mathrm{a}, \mathrm{b})$ described the principal biological characteristics of the parasite; these provided the basis of a dependable experimental infection system which allowed controlled studies of early stages of the hostparasite interaction (Rubio-Godoy \& Tinsley 2002). In the present study, fish were injected with crude parasite extracts prior to D. sagittata challenge to assess whether the attempted vaccination altered the outcome of controlled infections.

\section{MATERIALS AND METHODS}

Fish. Oncorhynchus mykiss fingerlings were purchased from a parasite-free hatchery. Batches of 30 to 50 fish were maintained in 5001 tanks at $13^{\circ} \mathrm{C}$ and fed $3.0 \mathrm{~mm}$ commercial feed pellets (Trout floating 30 ; BOCM Pauls). Animals were allowed at least $7 \mathrm{~d}$ to recover from transport-related stress before the experimental procedures.

Parasites. Rainbow trout naturally infected with Discocotyle sagittata from the Isle of Man were transported to Bristol and housed at $13^{\circ} \mathrm{C}$ under a light:dark regimen of 12:12 h. Parasite eggs were collected by filtration (125 $\mu \mathrm{m}$ nylon mesh sieve), washed in distilled water and incubated at $13^{\circ} \mathrm{C}$ under a $12: 12 \mathrm{~h}$ light:dark photoperiod. After embryonation (ca. 28 d), egg batches hatch en masse in the first hour of the dark period (Gannicott \& Tinsley 1997), allowing collection of recently emerged infective stages. Under the dissecting microscope, batches of 100 active oncomiracidia were collected with a Pasteur pipette and placed in Petri dishes; they were never allowed to reach an age $>2 \mathrm{~h}$ before use.

Infection procedure. Fish (ca. $20 \mathrm{~cm}$ long) were placed individually in infection tanks (ca. $11.5 \mathrm{l}, 13^{\circ} \mathrm{C}$ ) and exposed to 100 active oncomiracidia for $24 \mathrm{~h}$, the first $12 \mathrm{~h}$ of which were in darkness. Afterwards, they were kept in $500 \mathrm{l}$ tanks at $13^{\circ} \mathrm{C}$ for 1 mo. Experimentally infected fish were anaesthetised terminally with $0.05 \%$ MS222, bled by caudal vein puncture to determine antibody titres, weighed and measured. Gills were removed and examined under the dissecting microscope, and the number of parasites per gill arch was recorded. Parasites were grouped in different (categorical) developmental cohorts based on the number of pairs of clamps they had grown (Gannicott \& Tinsley 1997, 1998a,b). Age cohort data were transformed into continuous variables by calculating the mean number of pairs of clamps attained by worms recovered from individual fish (Rubio-Godoy \& Tinsley 2002). This average, the Developmental Index, was calculated as follows:

Developmental Index $=\frac{\left.\sum \text { (worms recovered } \times \text { developmental cohort }\right)}{\text { total number of worms }}$

Blood samples were allowed to clot overnight $\left(4^{\circ} \mathrm{C}\right)$ and sera were kept at $-70^{\circ} \mathrm{C}$ until analysed.

Immunization. A crude worm extract was prepared by adapting a method developed for Taenia spp. (Molinari et al. 1993). Briefly, whole frozen and formalinfixed worms were washed and homogenised in PBS $\mathrm{pH}$ 7.0. The homogenate was centrifuged (30 min, $3400 \times g$ ) and the supernatant filtered through $0.2 \mu \mathrm{m}$ Millipore membranes; this filtrate was subsequently called 'worm extract'. The centrifugation pellet was retained and further processed in parallel to the filtrate; this was subsequently referred to as 'pellet extract'. Both extracts were dialysed against PBS and lyophilised in sterile vials. The parasite 'egg extract' was prepared by homogenising embryonated eggs incubated for $2 \mathrm{wk}$ at $13^{\circ} \mathrm{C}$ (about halfway through embryo development) in PBS pH 7.0, and filtration through $0.2 \mu \mathrm{m}$ Millipore membranes. The antigenic preparations were kept at $-70^{\circ} \mathrm{C}$ until used and their total protein concentration was determined by the method of Lowry et al. (1951).

Anaesthetised fish were treated with $10 \mu \mathrm{g}$ (total protein concentration) each of worm, pellet or egg extract dissolved in $0.5 \mathrm{ml}$ PBS $\mathrm{pH} 7.0$ by intraperitoneal (i.p.) injection, without adjuvant. Groups immunized once were injected 2 wk before parasite challenge; those vaccinated twice were treated 4 and 2 wk or 6 and 4 wk prior to exposure. Control groups received i.p. PBS only and were infected during the same week's post-injection as immunized fish. One further group received $10 \mu \mathrm{g}$ pellet extract i.p. in $0.25 \mathrm{ml}$ PBS emulsified in an equal amount of Freund's complete adjuvant (FCA; Sigma Chemical) 4 wk prior to infection; controls for this group received i.p. FCA only and were challenged concurrently.

Immunological parameters. Ethanol-fixed DisCocotyle sagittata were sonicated on ice without the addition of anti-proteases (Ultrasonic Liquid Processor XL 2020; Heat System) and used as antigenic source. ELISA was performed in 96-well microtitre plates coated $\left(12 \mathrm{~h}\right.$ at $\left.5^{\circ} \mathrm{C}\right)$ with antigen $\left(5 \mu \mathrm{g} \mathrm{ml} \mathrm{m}^{-1}\right.$ protein) dissolved in $100 \mathrm{\mu l}$ coating buffer $(4.29 \mathrm{~g}$ $\mathrm{Na}_{2} \mathrm{CO}_{3} \cdot 10 \mathrm{H}_{2} \mathrm{O}$ and $2.93 \mathrm{~g} \mathrm{NaHCO}_{3}$ in $1 \mathrm{l}$ of distilled water, $\mathrm{pH}$ 9.6). Microtitre plates were then washed 5 times with washing buffer (PBS with $0.05 \%$ Tween20; Merck). Uncoated sites were blocked by adding $100 \mu$ l blocking buffer (PBS with $0.5 \%$ bovine serum albumin, BSA; Sigma Chemical) per well for $15 \mathrm{~min}$. Between the following steps, plates were washed 5 times with washing buffer. Sera were diluted serially 
(1:2 to $1: 1024$ ) in dilution buffer (PBS with $0.1 \%$ BSA) and $100 \mu \mathrm{l}$ of each dilution was added in duplicate to each of the coated wells and incubated for $1 \mathrm{~h}$ under gentle agitation. Rabbit anti-salmon Ig (Buchmann \& Pedersen 1994) (100 $\mu \mathrm{l}$ at 1:1000 in dilution buffer) was added to each well and incubated for $1 \mathrm{~h}$. Finally, $100 \mu \mathrm{l}$ peroxidase-conjugated goat anti-rabbit Ig (1:2000 in dilution buffer; Sigma Chemical) was supplied to each well. Following incubation for $1 \mathrm{~h}, 100 \mu \mathrm{l}$ enzyme substrate (o-phenylenediamine, OPD; Sigma Chemical) was added per well, and plates incubated for 10 to $15 \mathrm{~min}$. Enzymatic reaction was stopped by adding $50 \mu \mathrm{l} 3 \mathrm{M} \mathrm{HCl}$ per well, and optical density (O.D.) determined at $492 \mathrm{~nm}$ using an ELISA-reader (Multiscan RC, Type 351; Labsystems). Each plate included 2 controls: wells with all antibodies and substrates except sample material (primary antibodies) as negative controls (blanks), and sera obtained from naïve fish. Values greater than 2 times the (blank) background absorbance were considered to be positive. ELISA was validated by means of the O.D. readings obtained from naïve sera, which were the same in the different plates/assay lots.

Western blots. Electrophoresis was done using a commercially available electrophoresis system (NuPAGE; Novex). Proteins from Discocotyle sagittata were separated by a $10 \%$ polyacrylamide gel at neutral $\mathrm{pH}$ by applying $25 \mu \mathrm{g}$ total protein of the sonicated antigen in sample buffer to each well. All samples were run under non-reducing conditions using MOPS (3-[N-morpholino] propane sulfonic acid) running buffer $(200 \mathrm{~V}$ for $1 \mathrm{~h})$. Antigens run on SDS-PAGE were transferred to $0.45 \mu \mathrm{m}$ nitrocellulose membranes using a blotting module (Xcell 50II Mini-Cell Blot module; Novex) (30 V for $1 \mathrm{~h}$ ). The nitrocellulose membrane was then blocked for unspecific binding with blocking buffer (PBS pH 7.2 with 1\% skimmed milk powder) for $15 \mathrm{~min}$ after which the trout antiserum dilution (1:20) was added. Following incubation for $1 \mathrm{~h}$ the membrane was washed $3 \times 5$ min with washing buffer (PBS with $0.05 \%$ Tween-20) before addition of rabbit anti-salmon Ig (1:1000). After incubation for $1 \mathrm{~h}$ and the $3 \times 5$ min washes, horseradish peroxidase conjugated goat-anti-rabbit antibody (Sigma A-0545) (1:2000) was added to the membranes and incubated for $1 \mathrm{~h}$. After $3 \times 5 \mathrm{~min}$ of washing, the blot was developed using diamino-benzidine-tetrahydrochloride (DAB) (Sigma D-5905) in PBS with $\mathrm{H}_{2} \mathrm{O}_{2}$.

Statistical analysis. Data were analysed with the statistical package SPSS for Windows 10.0. One-way ANOVA (General Linear Models) was used to analyse the effect of immunization on numbers of worms recovered and on antibody titres in each treatment group, and to validate ELISA tests; the significance level was set at $p<0.05$. Tukey's test was used for post- hoc analysis. For each experimental group, the mean intensity (Bush et al. 1997) was calculated. As an arbitrary measure of efficacy, the percentage of the immunized population harbouring fewer parasites than the overall mean control intensity minus 1 SD was calculated; efficacy rates are only illustrative and were not used in any statistical calculations.

\section{RESULTS}

Fish measured $19.6 \pm 0.23 \mathrm{~cm}$ (mean $\pm \mathrm{SE}$ ) in length and weighed $96.6 \pm 3.24 \mathrm{~g}$; there was no significant difference between experimental $(\mathrm{n}=118)$ and control (n=104) fish (length $F_{1}=0.614, \mathrm{p}=0.434$; weight $F_{1}=$ $0.414, \mathrm{p}=0.521)$. In all trials, fish were dissected $1 \mathrm{mo}$ p.i. and all experimentally infected animals harboured parasites. Although worm numbers differed between treatment groups (see below), the Developmental Index calculated for each did not: 1 mo p.i. an overall mean of $1.53 \pm 0.07$ was found, which did not differ significantly between groups $\left(F_{1}=0.134, \mathrm{p}=0.715\right)$.

Treatment with 1 or 2 doses of $10 \mu \mathrm{g}$ worm or pellet extract applied in PBS resulted in most cases in significantly reduced worm burdens in immunized as compared with control fish. Table 1 shows the mean parasite intensities found in control and immunized groups, as well as the statistical probability and the illustrative, arbitrary efficacy rate calculated for each trial. As per definition, immunization was effective in $\geq 50 \%$ of fish receiving worm or pellet extract, since they exhibited intensities equal to or less than 37 worms per fish; overall, $16 \%$ of control fish had burdens in this range. In all but 1 trial (pellet, $2 \mathrm{wk}$ ), statistical analysis demonstrated that the protective effect of immunization suggested by the arbitrary efficacy rate was significant. Parasite intensities in egg extract-injected fish were not different from their respective controls. The degree of protection conferred by immunization did not increase with different application regimes nor boosting: despite differences in the arbitrarily calculated vaccination efficacies, mean intensities in immunized fish are comparable throughout (Table 1; post-hoc analysis showed no significant differences between groups).

Overall, worm extract immunization significantly reduced infection levels $\left(F_{1}=33.79 ; \mathrm{p}<0.0001\right)$, but neither the immunization regime ( 1 or 2 doses; different application schedules) $\left(F_{2}=0.381 ; \mathrm{p}=0.685\right)$ nor the interaction between regime and application of extract $\left(F_{2}=0.765 ; \mathrm{p}=0.469\right)$ significantly modified the result. A similar situation was found when pellet extract was applied in PBS: immunization significantly modified the infection outcome $\left(F_{1}=22.19 ; \mathrm{p}<0.0001\right)$ while neither regime $\left(F_{2}=2.77 ; \mathrm{p}=0.069\right)$, nor the regime $\times$ immunization interaction $\left(F_{2}=1.61 ; \mathrm{p}=\right.$ 
Table 1. Effect of Discocotyle sagittata extract immunization on parasite recovery 1 mo post-infection. FCA: Freund's complete adjuvant. Efficacy: percentage (and fraction) of immunized population harbouring fewer worms than overall control mean -1 SD, i.e. intensities equal to or less than 37 worms/host. ns: not significant

\begin{tabular}{|c|c|c|c|c|c|c|c|c|}
\hline \multicolumn{3}{|c|}{ Worm - } & \multicolumn{3}{|c|}{ Pellet } & \multicolumn{2}{|c|}{$\longrightarrow$ Egg -} & \multirow{2}{*}{$\begin{array}{c}\text { Pellet in FCA } \\
4 \mathrm{wk}\end{array}$} \\
\hline $2 \mathrm{wk}$ & $4-2 \mathrm{wk}$ & $6-4 \mathrm{wk}$ & $2 \mathrm{wk}$ & $4-2 \mathrm{wk}$ & $6-4 \mathrm{wk}$ & $2 \mathrm{wk}$ & $6-4 \mathrm{wk}$ & \\
\hline \multicolumn{9}{|l|}{ Efficacy } \\
\hline $67 \%(6 / 9)$ & $50 \%(9 / 18)$ & $67 \%(8 / 12)$ & $50 \%(6 / 12)$ & $75 \%(15 / 20)$ & $82 \%(9 / 11)$ & $0 \%(0 / 10)$ & $0 \%(0 / 10)$ & $56 \%(9 / 16)$ \\
\hline \multicolumn{9}{|c|}{ Control group; mean worms $/$ host $\pm S E(n)^{a}$} \\
\hline $\begin{array}{c}45.9 \pm 6.28 \\
(10)\end{array}$ & $\begin{array}{c}45.3 \pm 1.49 \\
(17)\end{array}$ & $\begin{array}{c}46.2 \pm 2.98 \\
(10)\end{array}$ & $\begin{array}{c}42.4 \pm 2.93 \\
(9)\end{array}$ & $\begin{array}{c}42.4 \pm 1.18 \\
(19)\end{array}$ & $\begin{array}{c}47.38 \pm 4.65 \\
(8)\end{array}$ & $\begin{array}{c}41.6 \pm 4.25 \\
(8)\end{array}$ & $\begin{array}{c}39.3 \pm 1.33 \\
(7)\end{array}$ & $\begin{array}{c}43.4 \pm 2.97 \\
(16)\end{array}$ \\
\hline \multicolumn{9}{|c|}{ Immunized group; mean worms/host \pm SE (n) } \\
\hline $\begin{array}{c}32.0 \pm 3.19 \\
(9)\end{array}$ & $\begin{array}{c}36.9 \pm 2.13 \\
(18)\end{array}$ & $\begin{array}{c}34.9 \pm 2.32 \\
(12)\end{array}$ & $\begin{array}{c}37.6 \pm 4.12 \\
(12)\end{array}$ & $\begin{array}{c}31.1 \pm 1.89 \\
(20)\end{array}$ & $\begin{array}{c}29.0 \pm 3.92 \\
(11)\end{array}$ & $\begin{array}{c}38.0 \pm 2.76 \\
(10)\end{array}$ & $\begin{array}{c}40.5 \pm 3.44 \\
\quad(10)\end{array}$ & $\begin{array}{c}36.2 \pm 3.47 \\
(16)\end{array}$ \\
\hline $\mathbf{p}$ & & & & & & & & \\
\hline 0.001 & 0.003 & 0.007 & ns & $<0.0001$ & 0.008 & ns & ns & 0.026 \\
\hline
\end{tabular}

0.206), significantly affected the results. The application of pellet extract in Freund's complete adjuvant (FCA) 1 mo prior to infection resulted in significant partial protection in $>50 \%$ of immunized fish (Table 1 ). However, the results obtained with adjuvanted pellet extract were comparable to those from fish that received 1 dose of pellet extract in PBS $\left(F_{1}=0.068 ; \mathrm{p}=\right.$ 0.797). Control fish that received FCA and no extract likewise had similar parasite intensities to control fish receiving saline only $\left(F_{1}=0.048 ; \mathrm{p}=0.829\right)$.

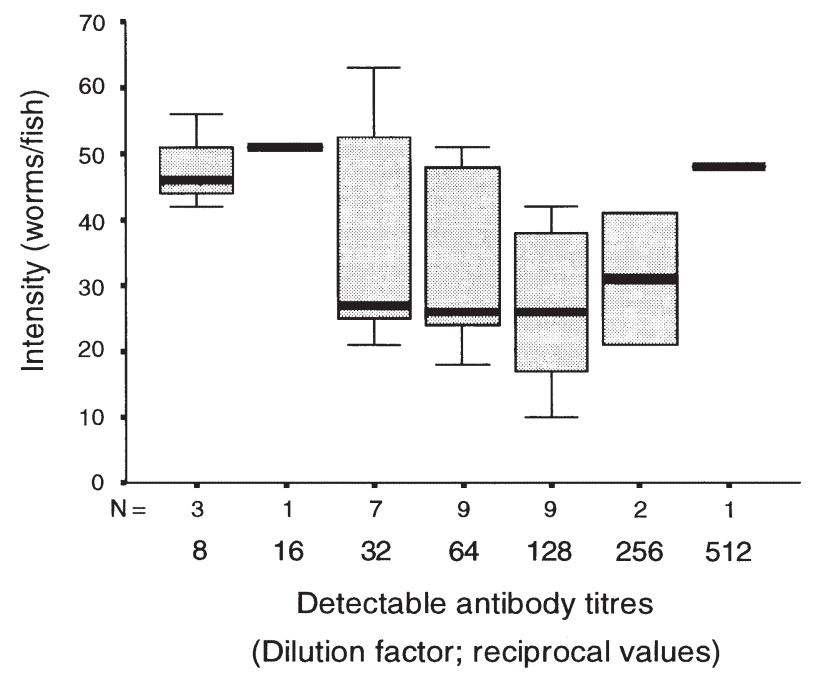

Fig. 1. Correlation between Discocotyle sagittata intensity and antibody titres at the time of rainbow trout Oncorhynchus mykiss dissection. Figure illustrates the median (thick line), the interquartile range containing $50 \%$ of the values (bar) and the range (whiskers) for each dilution factor
ELISA was used to determine the mean anti-Discocotyle sagittata antibody titres at the time of dissection of experimentally infected and naïve fish, which corresponds to $6 \mathrm{wk}$ post injection for fish immunized once, and 8 to $10 \mathrm{wk}$ post first-injection for those immunized twice. Titres were expressed as the 2-fold dilution factor beyond which no response was detected. Eight control and 16 immunized serum samples from each worm and pellet in PBS extract-injected groups were selected at random for immunological analysis. Naïve serum gave positive responses up to a mean dilution factor $( \pm \mathrm{SE})$ of $3.71 \pm 0.34$, control serum of $5.06 \pm 0.14$ and immunized serum of $6.00 \pm 0.24$; statistical analysis validated the ELISA by showing the naïve serum O.D. readings in all plates were comparable, indicating that immunization significantly modified detectable antibody titres $\left(F_{2}=8.601 ; \mathrm{p}<0.0001\right)$, and post-hoc tests showed the 3 groups were significantly different. Moreover, a significant negative correlation was found between the number of parasites recovered from immunized fish (pooled worm- and pellet-extract immunized groups) and antibody titres, expressed as 2-fold dilution factors $\left(\mathrm{R}^{2}=0.866 ; F_{6}=26.997 ; \mathrm{p}<0.0001 ;\right.$ Fig. 1$)$.

Western blot results indicated extract-immunized fish recognise 6 to 8 antigenic bands with different molecular sizes (Fig. 2): worm extract, ca. 100, 50, 45, 40, 38, 30, 20 and $11 \mathrm{kDa}$; pellet extract, ca. 126, 50, 30, 18,16 and $4 \mathrm{kDa}$. There is however a clear difference in recognition intensity: pellet extract-immunized sera reacted mainly against a ca. $30 \mathrm{kDa}$ band, while worm extract-immunized sera primarily recognised antigens ca. $38 \mathrm{kDa}$ in size. Sera from infected control fish also reacted faintly against antigenic bands ca. 100, 50, 38, 30 and $4 \mathrm{kDa}$ in size. 


\section{DISCUSSION}

The present experiments demonstrate that active immunization of rainbow trout with Discocotyle sagittata extracts confers partial protection against infection. As reviewed by Buchmann et al. (Buchmann 1999, Buchmann et al. 2001, Buchmann \& Lindenstrøm 2002), a number of investigations have shown that teleost fishes are capable of mounting protective immune responses to monogenean parasites, which suggests that vaccination is possible. However, successful immunization has been achieved primarily against some bacterial and viral diseases (Nakanishi \& Ototake 1997, Palm et al. 1998), and effective vaccines against helminths have proved mainly elusive. For instance, no protective immunity resulted from the injection of fish with sonicated Neobenedenia girellae (Bondad-Reantaso et al. 1995), nor with whole Gyrodactylus alexanderi (Lester 1974). However, partial protection was achieved in carp following immunization with dactylogyrids (Vladimirov 1971), and in rockfish Sebastes schlegeli vaccinated against the gillinhabiting monogenean Microcotyle sebastis (Kim et al. 2000). The availability of a dependable experimental infection system with the polyopisthocotylean $D$. sagittata prompted the assessment of the role of immunity in controlling parasite burdens. Experimental exposure of rainbow trout to $D$. sagittata resulted in $100 \%$ prevalence and ca. $50 \%$ infection efficiency up to 3 mo p.i. (Rubio-Godoy \& Tinsley 2002). This is in contrast with the usually low prevalence and intensity characteristics of natural monogenean infections. However, experimental, 3 mo old D. sagittata infection may represent only the early stages of the host-parasite interaction, before immunity controls worm burdens effectively. Long-term field- and lab-based studies with polystomatid monogeneans would support this hypothesis: in both scenarios, very efficient transmission rates lead to high intensities of juvenile stages, but parasite numbers undergo a marked attrition between invasion and maturity (Tinsley \& Jackson 2002). Host immunity has been proposed to play a major role in regulating invading polystomatid juvenile numbers before they can contribute to transmission (Tinsley 1995, 1999, Tinsley \& Jackson 2002). In fish-monogenean interactions, both non-specific and specific immune mechanisms have been proposed to protect fish against pathogens. Non-specific responses have been implicated in the control of gyrodactylid monogeneans, particularly complement (Buchmann 1998, Harris et al. 1998) and leucocytes (Buchmann \& Bresciani 1999). Antibodies and various other immune compounds in rainbow trout mucus have been reported on several occasions (St Louis-Cormier et al. 1984, Buchmann \& Bresciani 1998) and these could be involved in host defences (Buchmann 1999, Buchmann \& Lindenstrøm 2002). G. derjavini distribution on rainbow trout exhibits a significant negative correlation with superficial mucous cell density, suggesting mucus may have an important effect on parasite site selection and infection dynamics (Buchmann \& Bresciani 1998).

The present work suggests immunization against Discocotyle sagittata is feasible and that specific antibodies are 1 of the several possible factors controlling parasite numbers, since partial protection correlated significantly with increased serum immuno-globulin titres as determined at the time of dissection. However, no simple cause-effect relationship can be proposed for several reasons. First, no serum samples were obtained at the time of infection nor prior to dissection, therefore no direct inference can be made of their putative protective role during invasion and the early stages of parasite establishment. Moreover, it is uncertain if antibody levels at the time of infection were relevant in the groups immunized once, since only $2 \mathrm{wk}$ had elapsed prior to challenge; but the possibility exists that antibodies are detected as soon as $10 \mathrm{~d}$ postimmunization in rainbow trout kept at 14 to $16^{\circ} \mathrm{C}$ (Palm et al. 1998). Second, no tests were performed to detect immunoglobulin (nor any other immune components) in mucus, which might be another route through which this gill-inhabiting parasite could be exposed to immune factors. Third, it is unlikely that immunoglob-

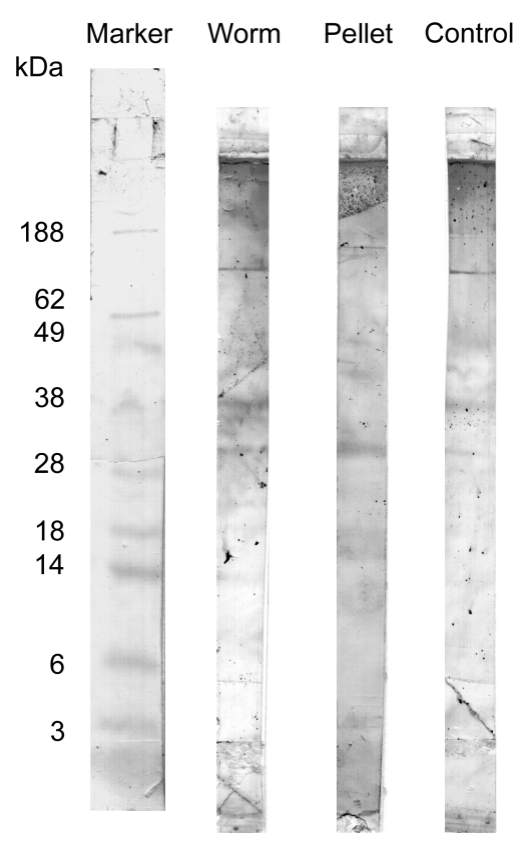

Fig. 2. Western blots of Discocotyle sagittata antigens revealed with sera from worm and pellet-extract immunized, and infected control rainbow trout Oncorhynchus mykiss. Sera were obtained at the time of dissection; i.e. at least $6 \mathrm{wk}$ post-immunization with parasite extracts 
ulin by itself is able to eliminate parasites, hence the lack of sterile immunity observed even in fish with relatively high antibody titres. And finally, the injection of parasite materials is likely to elicit inflammatory reactions, which could account for the reduced burdens recorded. Nevertheless, the specific, probably noninflammatory nature of the non-sterile immunity observed is suggested by the fact that neither egg extract nor FCA alone modified infection outcome significantly. While it is uncertain whether embryonated eggs contain effective immunogens, and therefore induce non-specific responses, FCA is not only an immunization adjuvant but is itself a powerful elicitor of non-specific immunity (Anderson 1992, Secombes 1994).

As reported previously (Rubio-Godoy \& Tinsley 2002), parasite intensity 1 mo p.i. in control fish was comparable in all groups, and reflected an infection efficiency of ca. $45 \%$. Interestingly, mean intensities in immunized fish were similar irrespective of the distinct vaccination schemes applied to different experimental groups. Thus, dissimilar immunization regimes resulted in comparable degrees of protection, both in terms of the proportion of the population showing reduced worm burdens and in the mean intensities recorded. This suggests boosting does not apparently result in increased immunity. The comparable effect of worm- and pellet-extract immunization is striking considering the 2 parasite extracts primarily induced recognition of different antigens (Fig. 2). Overall, immunization seemed to induce the transition between 2 equilibria, rather than progressively increase protection and correspondingly decrease parasite burdens. As shown in Fig. 1, fish with antibody titres of $2^{4}$ (dilution factor 16) or less (including naïve fish not depicted in Fig. 1) have mean (and median) parasite intensities consistently around 45 worms per host, which could be considered a 'permissive equilibrium parasite intensity'. In contrast, past an antibody titre of $2^{5}$ (dilution factor 32) mean intensities abruptly fall to ca. 34 worms per host (and slightly lower medians), which could be described as a distinct 'immune equilibrium parasite intensity'. These 2 hypothetical states would be analogous to a separation of the immunized population into responding and non-responding individuals, as typically subdivided in clinical trials. In this study, $64 \%$ of fish could be considered to have responded to immunization, based on the arbitrary definition of efficacy relating to infection levels. Responder fish had higher mean antibody titres than both controls and immunized non-responders, but this difference was only significant for control fish (data not shown). Despite the non significant difference of antibody titres between responder and non-responder fish, it is interesting that elevated immunoglobulin levels tended to correlate with increased protection. In particular, fish with antibody titres $>1: 32$ consistently harboured reduced parasite burdens, and these constituted the bulk of the immunized population irrespective of the antigenic extract employed (Fig. 1). Thus, it would be reasonable to argue that immunization shifted the balance between the 2 parasite intensities (and hypothetical equilibria) observed, suggesting that host responses are among the several possible factors which ultimately result in an overdispersed distribution. The occurrence of 2 distinct equilibria in this report would be analogous to Discocotyle sagittata infection levels found in field studies, in which a proportion of fish exposed to long-term transmission exhibit very high intensities, while others raised in the same conditions (and ponds) present low burdens (Gannicott 1997).

The actual host factors limiting parasite numbers are not known, but given the negative correlation found in this study between antibody titres and worm burdens, it would be tempting to propose that immunoglobulin accounts for the partial protection observed. The evidence, however, is not clear-cut. Naïve fish exhibited detectable antibody titres against the sonicated parasite; this positive response may be due to naturally occurring antibodies with low affinity to Discocotyle sagittata. Comparable antibody titres in control and immunized fish suggest that infection elicits a certain degree of humoral immunity, detectable 1 mo p.i. Moreover, naturally infected fish exhibit very high anti-D. sagittata antibody levels (author's unpubl. result), which do not seem to confer effective protection against either established or invading parasites. Similarly, other helminth infections result in increased antibody levels which do not provide effective immunity, as is the case for Schistosoma mansoni (MacDonald et al. 2002), or even seem to correlate with susceptibility, as found for Taenia solium (de Aluja et al. 1999). Prime candidates as effector molecules or cells are those that increase in production or number with an infection (Viney 2002), but in most cases the actual effector mechanisms confering resistance remain unsolved. Arguably, protective mechanisms are combinatorial, encompassing non-specific and cellular factors, as well as humoral components. In fish, defence mechanisms against parasites include, in addition to immunoglobulin, complement and other non-specific factors such as lectins, acute phase reactants, lysozyme and antimicrobial peptides, as well as several cell types (Woo 1992, Buchmann \& Lindenstrøm 2002).

Antibodies have been detected against some monopisthocotylean monogeneans: for instance against dactylogyrids (Vladimirov 1971) and pseudodactylogyrids (Buchmann 1997, Mazzanti et al. 1999). The differential susceptibility to the nematode Anguillicola crassus of Japanese eel Anguilla japonica, compared 
with the European eel A. anguilla has been correlated to antibody titres (Nielsen 1999). Similarly, this work presents evidence of increased antibody titres in partially protected fish. However, considering naïve trout also had detectable antibody levels, further studies are needed to elucidate whether, or to what extent, immunoglobulin alone or in combination with other factors affects the integrity of Discocotyle sagittata.

Acknowledgements. We are grateful to Prof. J. L. Molinari (UNAM, Mexico) for advice on the preparation of the parasite extracts. We thank J. Ballard and K. Jervis for access to fish in the Isle of Man. M.R.G. is supported by CONACYT (Mexico) \& ORS (UK) postgraduate scholarships.

\section{LITERATURE CITED}

Anderson DP (1992) Immunostimulants, adjuvants, and vaccine carriers in fish: applications to aquaculture. Annu Rev Fish Dis 2:281-307

Bondad-Reantaso MG, Ogawa K, Yoshinaga T, Wakabayashi $\mathrm{H}$ (1995) Acquired protection against Neobenedenia girellae in Japanese flounder. Fish Pathol 30:233-238

Buchmann K (1997) Infection biology of gill parasitic monogeneans with special reference to the congeners Pseudodactylogyrus bini and P. anguillae (Monogenea: Platyhelminthes) from European eel. DVSc thesis, Royal Veterinary and Agricultural University Frederiksberg

Buchmann K (1998) Binding and lethal effect of complement from Oncorhynchus mykiss on Gyrodactylus derjavini (Platyhelminthes: Monogenea). Dis Aquat Org 32: 195-200

Buchmann K (1999) Immune mechanisms in fish skin against monogeneans - a model. Folia Parasitol 46:1-9

Buchmann K, Bresciani J (1998) Microenvironment of Gyrodactylus derjavini on rainbow trout Oncorhynchus mykiss: association between mucous cell density in skin and site selection. Parasitol Res 84:17-24

Buchmann K, Bresciani J (1999) Rainbow trout leucocyte activity: influence on the ectoparasitic monogenean Gyrodactylus derjavini. Dis Aquat Org 35:13-22

Buchmann $K$, Lindenstrøm $T$ (2002) Interactions between monogenean parasites and their fish hosts. Int J Parasitol 32:309-319

Buchmann K, Pedersen K (1994) A study on teleost phylogeny using specific antisera. J Fish Biol 45:901-903

Buchmann K, Lindenstrøm T, Bresciani J (2001) Defence mechanisms against parasites in fish and the prospect for vaccines. Acta Parasitol 46:71-81

Bush AO, Lafferty KD, Lotz JM, Shostak AW (1997) Parasitology meets ecology on its own terms: Margolis et al. revisited. J Parasitol 83:575-583

de Aluja AS, Villalobos ANM, Plancarte A, Rodarte LF, Hernandez M, Zamora C, Sciutto E (1999) Taenia solium cysticercosis: immunity in pigs induced by primary infection. Vet Parasitol 81:129-135

Gannicott AM (1997) The biology of Discocotyle sagittata (Monogenea) infecting trout. PhD thesis, University of Bristol

Gannicott AM, Tinsley RC (1997) Egg hatching in the monogenean gill parasite Discocotyle sagittata from the rainbow trout (Oncorhynchus mykiss). Parasitology 114: 569-579

Gannicott AM, Tinsley RC (1998a) Larval survival character- istics and behaviour of the gill monogenean Discocotyle sagittata. Parasitology 117:491-498

Gannicott AM, Tinsley RC (1998b) Environmental effects on transmission of Discocotyle sagittata (Monogenea): egg production and development. Parasitology 117:499-504

Harris PD, Soleng A, Bakke TA (1998) Killing of Gyrodactylus salaris (Platyhelminthes, Monogenea) mediated by host complement. Parasitology 117:137-143

Jahn TL, Kuhn LR (1932) The life history of Epibdella melleni MacCallum 1927, a monogenetic trematode parasitic on marine fishes. Biol Bull 62:89-111

Kim KH, Hwang YJ, Cho JB, Park SI (2000) Immunization of cultured juvenile rockfish Sebastes schlegeli against Microcotyle sebastis (Monogenea). Dis Aquat Org 40: 29-32

Lester RJG (1974) Attachment of Gyrodactylus to Gasterosteus and host response. J Parasitol 58:717-722

Lowry OH, Rosenbrough NJ, Farr AL, Randall RJ (1951) Protein measurement with the Folin phenol reagent. J Biol Chem 193:265-275

MacDonald AS, Araujo MI, Pearce EJ (2002) Immunology of parasitic helminth infections. Infect Immunol 70:427-433

Mazzanti C, Monni G, Varriale AMC (1999) Observations on antigenic activity of Pseudodactylogyrus anguillae (Monogenea) on the European eel (Anguilla anguilla). Bull Eur Assoc Fish Pathol 19:57-59

Molinari JL, Soto R, Tato P, Rodríguez D, Retana A, Sepúlveda J, Palet A (1993) Immunization against porcine cysticercosis in an endemic area in Mexico: a field and laboratory study. Am J Trop Med Hyg 49:502-512

Nakanishi T, Ototake M (1997) Antigen uptake and immune responses after immersion vaccination. In: Gudding $\mathrm{R}_{\mathrm{r}}$ Lillehaug A, Midtlyng PJ, Brown F (eds) Fish vaccinology. Developments in biological standardization, Vol 90. Karger, Basel, p 59-68

Nielsen ME (1999) An enhanced humoral immune response against the swimbladder nematode, Anguillicola crassus, in the Japanese eel, Anguilla japonica, compared with the European eel, A. anguilla. J Helminthol 73:227-232

Nigrelli RF (1937) Further studies on the susceptibility and acquired immunity of marine fishes to Epibdella melleni, a monogenetic trematode. Zoologica 22:185-192

Nigrelli RF, Breder CM (1934) The susceptibility and immunity of certain marine fishes to Epibdella melleni, a monogenetic trematode. J Parasitol 20:259-269

Palm RC, Landolt ML, Busch RA (1998) Route of vaccine administration: effects on the specific humoral response in rainbow trout Oncorhynchus mykiss. Dis Aquat Org 33: $157-166$

Rubio-Godoy M, Tinsley RC (2002) Trickle and single infection with Discocotyle sagittata (Monogenea: Polyopisthocotylea): effect of exposure mode on parasite abundance and development. Folia Parasitol 49:269-278

Scott ME (1985) Dynamics of challenge infections of Gyrodactylus bullatarudis Turnbull (Monogenea) on guppies, Poecilia reticulata (Peters). J Fish Dis 8:495-503

Scott ME, Robinson MA (1984) Challenge infections of Gyrodactylus bullatarudis (Monogenea) on guppies (Poecilia reticulata) following treatment. J Fish Biol 24:581-586

Secombes CJ (1994) Enhancement of fish phagocyte activity. Fish Shellfish Immunol 4:421-436

Secombes CJ, Chappell LH (1996) Fish immune responses to experimental and natural infection with helminth parasites. Annu Rev Fish Dis 6:167-177

Slotved HC, Buchmann K (1993) Acquired resistance of the eel, Anguilla anguilla L., to challenge infections with gill monogeneans. J Fish Dis 16:585-591 
St Louis-Cormier EA, Osterland CK, Anderson PD (1984) Evidence for a cutaneous secretory immune system in rainbow trout (Salmo gairdneri). Dev Comp Immunol 8: 71-80

Thoney DA, Hargis WHJ (1991) Monogenea (Platyhelminthes) as hazards for fish in confinement. Annu Rev Fish Dis 1:133-153

Tinsley RC (1995) Parasitic disease in amphibians: control by the regulation of worm burdens. Parasitology 111:S153-178

Editorial responsibility: Wolfgang Körting, Hannover, Germany
Tinsley RC (1999) Parasite adaptations to extreme conditions in a desert environment. Parasitology 119:S31-56

Tinsley RC, Jackson JA (2002) Host factors limiting monogenean infections: a case study. Int J Parasitol 32:353-365

Viney ME (2002) How do host immune responses affect nematode infections? Trends Parasitol 18:63-66

Vladimirov VL (1971) The immunity of fishes in the case of dactylogyrosis. Parasitologiya 5:51-58 (in Russian)

Woo PTK (1992) Immunological responses of fish to parasitic organisms. Annu Rev Fish Dis 2:339-366

Submitted: April 30, 2002; Accepted: February 2, 2003

Proofs received from author(s): May 28, 2003 